\title{
Posterior thoracic segmental pedicle screw instrumentation: Evolving methods of safe and effective placement
}

\author{
S. C. Zeiller, J. Lee, M. Lim, A. R. Vaccaro \\ Thomas Jefferson University, Philadelphia, PA, USA
}

\begin{abstract}
The use of pedicle screw instrumentation in the spine has evolved over the last two decades. The initial use of pedicle screws began in the lumbar spine. As surgeons have become more comfortable with the complex anatomy required for accurate screw placement, the use of pedicle instrumentation has evolved to include their use in the thoracolumbar and thoracic spine. The impetus behind their increased use is a result of the many advantages that pedicle screw anchorage offers over traditional hook and rod constructs. Improved deformity correction and overall construct rigidity are two important advantages of pedicle screw instrumentation due its three-column control over the spinal elements. First, pedicle screw instrumentation obviates the need to place instrumentation within the spinal canal with its inherent risk of neurologic injury. Second, the placement of pedicle screws is independent of facet or laminar integrity and thus has been extremely useful in traumatic, neoplastic, and degenerative conditions. The benefits of pedicle screws in the thoracic spine has been tempered by the potential for catastrophic neurological or soft tissue injuries due to the close proximity of these structures. The narrow and inconsistent shape of the thoracic pedicles, especially in spinal deformity, makes their placement technically challenging. As a result, surgeons have employed a number of techniques to ensure the safe and efficacious placement of thoracic pedicle screws. Detailed anatomic landmarks used to determine pedicle location, intraoperative imaging including navigation, and neurophysiological monitoring are some of the techniques currently used by surgeons. The implementation of these techniques and a thorough understanding of the complex three-dimensional anatomy have allowed surgeons to successfully place thoracic and thoracolumbar pedicle screws.
\end{abstract}

Key words: Computerized tomography; safety; spinal fixation; thoracic pedicle screw
The techniques of pedicle screw placement in the spine has evolved considerably over the last two decades. Pedicle screw instrumentation was initially popularized in the lumbar spine. Through detailed cadaveric studies and clinical experience surgeons have become familiar with the complex three-dimensional (3D) anatomy involved in safely placing lumbar pedicle screws. Pedicle screw anchorage of the 'force nucleus' or pedicle, allows improved deformity correction and rigid stability when compared to hook and rod fixation due to the three-column control pedicle screw fixation affords. ${ }^{[1,2]}$ As surgeons became more comfortable with lumbar pedicle screw instrumentation, a slow progression of their use in the thoracolumbar region and more cephalad has ensued over the last five to 7 years.

Pedicle screw placement in the thoracic and thoracolumbar spine has many advantages. The use of pedicle screws obviates the need for placement of any instrumentation within the spinal canal. This is particularly important when docking a rod to the screws, which, in the case of rod-hook anchorage, may inadvertently advance a supralaminar or sublaminar hook into the spinal canal. Pedicle screw anchorage is independent of facet and laminar integrity and is extremely useful in cases of trauma or a previous posterior decompression. Finally, the increased biomechanical strength of pedicle screw fixation allows, in most clinical situations, fewer segmental levels to be incorporated into the fusion.

The advantages of pedicle screw instrumentation in the thoracic spine have been tempered by the concern for the safety and accuracy associated with their placement. The close proximity of the spinal cord and major soft tissue structures including the aorta, esophagus, and lung, makes suboptimal placement a concern, especially on the left side of the spine due to the posterior lateral position of the aorta. ${ }^{[3]}$ In addition, cannulating the narrow and inconsistent shape of the thoracic pedicles especially in deformity has proven to be technically challenging. Since the safety and efficacy of thoracic pedicle screws is dependent on their proper placement within the spine, several techniques have evolved to ensure that this occurs. The objective of this paper is to review 
current as well as evolving techniques and principles associated with the safe and efficacious placement of thoracic and thoracolumbar pedicle screws.

\section{Preoperative radiologic workup}

The preoperative radiographic workup is the critical first step in preparing for placement of thoracic and thoracolumbar pedicle screws. AP and lateral plain radiographs illustrate global thoracic spinal alignment and a rough estimation of pedicle size (AP view) and unusual anatomic variants. Preferably these films are taken on a 36 in. cassette. This enables the surgeon to evaluate overall spinal coronal and sagittal alignment. Plain radiographs also become a useful template to record the proposed operative approach with a radiographic crayon along with any notes on unique patient characteristics, such as anatomic variants, as these imaging studies routinely accompany the patient into the operating theatre.

Advanced imaging modalities such as CT and MRI provide valuable information on pedicle morphometry and neural anatomy. Interestingly, as surgeons have become more comfortable with thoracic pedicle screw fixation, advanced imaging studies are being used less frequently in the presurgical planning stages when imaging of the neural elements is not necessary. For surgeons less familiar with thoracic pedicle screw insertion, transaxial CT imaging will demonstrate pedicle size, trajectory, and the proximity of the aorta to the lateral pedicle boundary. Advanced imaging may also assist in pedicle screw diameter and length selection, as well as anticipated screw insertion trajectory (Figure 1). In traumatic or pathologic disorders such as infection or tumor, advanced imaging studies may assist in vertebral level instrumentation selection and assist in determining which pedicles should be excluded due to pathologic compromise.

The most important contribution of advanced imaging technology in regards to thoracic pedicle fixation is identifying the location of the aorta in relationship to the thoracic pedicle

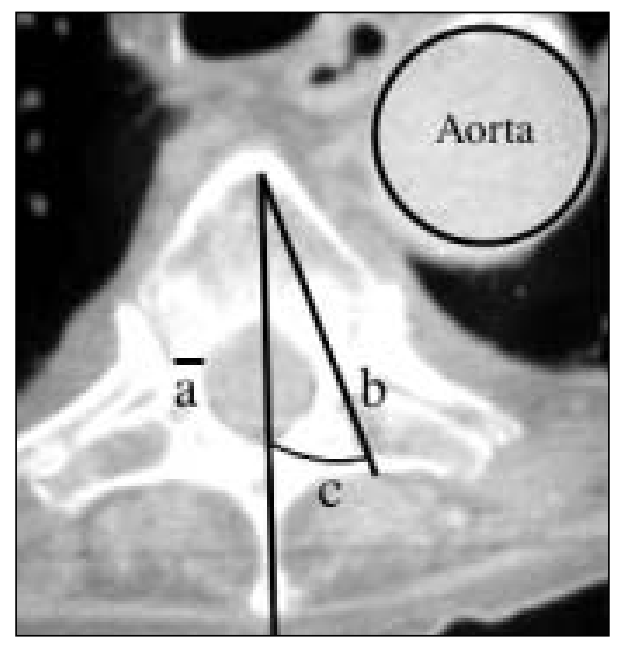

Figure 1: Axial CT image of a thoracic vertebra illustrating pertinent preoperative measurements. (a) width of pedicle, (b) potential length of screw and (c) transverse screw angle and pedicle screw starting point. Typically the thoracic aorta is located in the left hemithorax anterolaterally with respect to the vertebral body. However, the position of the aorta is markedly different in patients with idiopathic scoliosis presenting with a primary right-thoracic curve compared to a patient without spinal deformity (Figure 2). Sucato and Duchene evaluated 36 patients with idiopathic scoliosis and a right thoracic curve and compared the position of the aorta to 43 patients without a spinal deformity. ${ }^{[4]}$ The position of the aorta in patients with a primary right thoracic curve was posterior and lateral compared to their nonscoliotic counterparts. The awareness of this position becomes critical when placing thoracic pedicle screws along the concavity of the spinal curve in a patient with a right-sided thoracic curve.

\section{Surgical exposure}

The patient is placed prone on a radiolucent four-poster frame or Jackson table to facilitate intraoperative imaging, maintain adequate sagittal alignment and minimize any pressure to the anterior thorax or abdomen. During soft tissue exposure, the patient can be pharmacologically paralyzed to facilitate soft tissue dissection and release, thus minimizing blood loss. Paralysis is then reversed during pedicle screw preparation in order not to interfere with electrophysiologic monitoring (motor-evoked potentials, EMG). The soft tissue dissection should be carried out to the tips of the transverse processes and should include defining clearly the lateral border of the pars interarticularis. It is imperative to clear all soft tissue from the bony anatomy so that the anatomic landmarks can be clearly defined and a solid arthrodesis can be achieved following decortication. Once the bony landmarks are clearly defined, pedicle screw preparation may begin.

\section{Free-hand technique}

The free-hand technique for placement of thoracic and thoracolumbar pedicle screws relies completely on using visible as well as palpable anatomic landmarks for accurate pedicle screw placement. This technique has been popularized by Lenke et $\mathrm{a}^{\left[{ }^{[5]}\right.}$ following their vast experience with thoracic and thoracolumbar pedicle screw placement. It is dependent on clearly defining the posterior element bony landmarks including the lateral border of the pars interarticularis, the entire transverse process and cephalad facetjoint. A recommended pedicle screw starting point has been suggested for all levels between T1 and T12. Beginning distally in the thoracic spine at T10-T12 the starting point is located at the junction of a vertical line along the lateral pars boundary and a transverse line dividing the transverse process in half. As you move proximally toward the midthoracic spine, the starting point begins to move medially. At T7-T9 the starting point is the most medial located along a vertical line just lateral to the midpoint of the superior articular process at a transverse location along the superior border of the transverse process (Figure 3). More proximally, the screw starting point again moves more laterally. At T1-T2 the starting point is located at the intersection of a vertical line along the lateral border of the pars interarticularis and a transverse line bisecting the transverse process. 

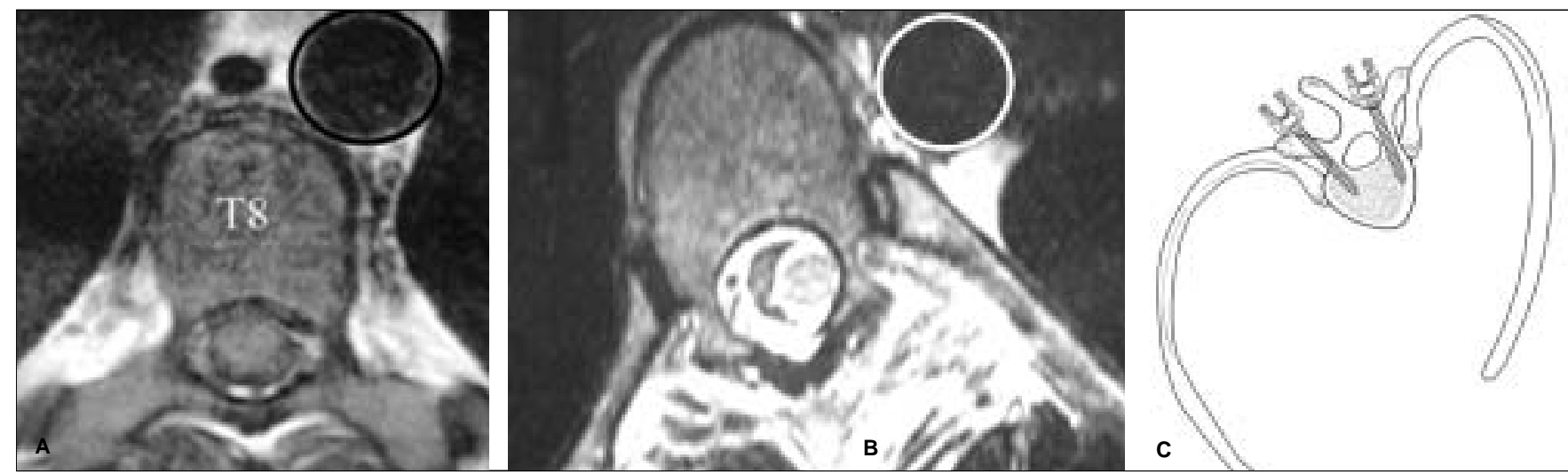

Figure 2: (A) Axial MRI image demonstrating the normal position of the aorta relative to the thoracic vertebra, (B) axial MRI image demonstrating position of aorta in a patient with primary right thoracic curve and (C) illustration representing the degree of rotation and pedicle deformity seen in setting of patients with thoracic scoliosis (courtesy of K2 Medical, Leesburg, VA)

Once the starting point has been established, a very clear and methodical series of steps has been described by Lenke et al. ${ }^{[5]} \mathrm{A}$ high-speed burr is used to penetrate the outer cortex over the pedicle entry point. Next, either a curved Lenke gearshift or a straight curette (3-0 cervical) is used to probe or mature the intended screw path within the pedicle. When using the curved Lenke gearshift, the curved tip should be facing the surgeon during the initial $20 \mathrm{~mm}$ of the pedicle path to minimize the potential for canal penetrance. Once the neurocentral junction of the pedicle is reached (typically at a depth of $20 \mathrm{~mm}$ ) the gearshift is removed and reinserted with the curved tip facing away from the surgeon. Positioning the pedicle probe tip medially assists in guiding the probe medially within the vertebral body. Next, the pedicle tract is palpated with a ball-tipped probe to verify the presence of a bony floor and an intact four-wall boundary. If a violation of bony integrity is noted at this point, redirecting the gearshift may be necessary in order to assure safe screw placement. The pedicle path is then tapped (preferably undertapped by $0.5-1.0 \mathrm{~mm}$ compared to the diameter of the selected screw) and the tract is repalpated with the ball-tipped probe to detect for any bony breaches. The pedicle screw is now inserted. Following screw

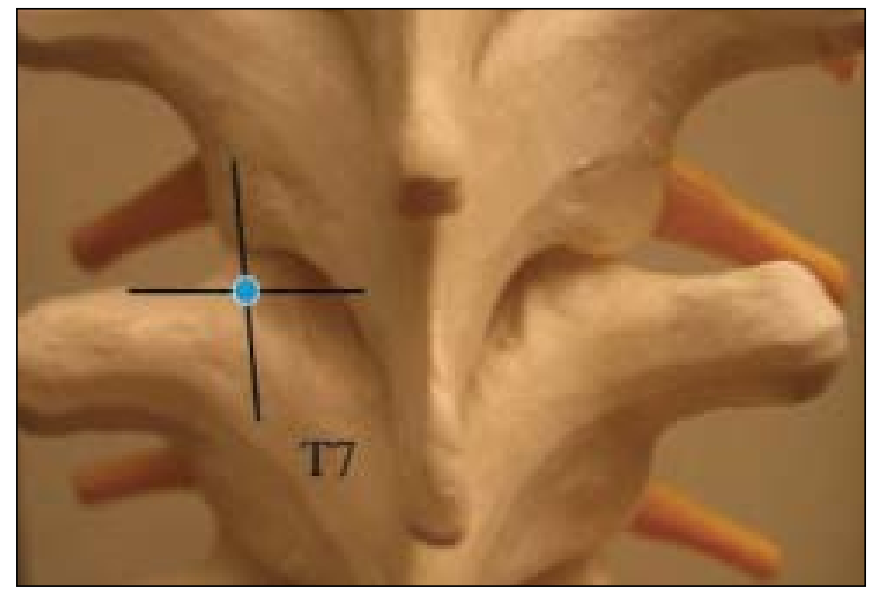

Figure 3: The starting point for the T7 pedicle is just lateral to the midpoint of the superior articular process and the superior aspect of the transverse process insertion, intraoperative imaging is then performed to verify acceptable screw positioning. Triggered EMG testing may be used to evaluate for proper lower thoracic screw placement (T8-T12), while motor-evoked potential (MEP) monitoring assists in monitoring spinal cord function for all thoracic levels instrumented. Once all screws are placed and the applicable screws have been tested via triggered EMG the rod can be docked to complete the construct (Figure 4).

\section{Penfield outside-in technique (Vaccaro)}

The senior author (A.R.V.) finds it useful to burr level the lateral border of prominent thoracic transverse processes to allow ease of screw insertion. A dorsally protruding transverse process has a tendency to push the surgeons hand medially during pedicle screw hole preparation and also interferes with screw seating by early

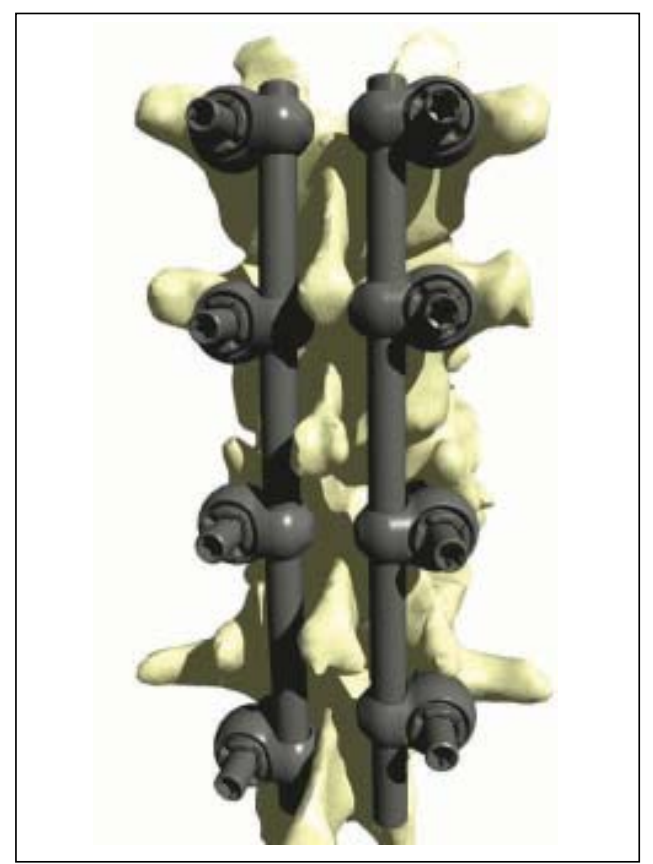

Figure 4: A completed thoracic stabilization procedure utilizing thoracic pedicle screws as the sole spinal anchor (courtesy of Life Spine, Hoffman Estates, IL) 
contact with the lateral screw tulip head. This also allows for a more lateral screw entry point for surgeons who prefer greater medial screw trajectory angulation to ensure vertebral body penetrance (Figure 5).

Burring the transverse process flush with the proposed pedicle screw starting point offers one more advantage. A Penfield \#4 can now easily palpate the lateral border of the superior articular process at its junction with the transverse process. This represents approximately the lateral border of the respective thoracic pedicle. The technique of palpating the pedicle from the 'outside-in' then allows the surgeon to gently, depending on the thoracic level, angle the pedicle probe medially during cannulation.

\section{Intraoperative imaging}

Intraoperative imaging techniques are useful in assisting safe screw path navigation. The most common imaging tools at this time are plain radiographs and multiplanar fluoroscopy. Advanced imaging modalities such as 3D fluoroscopy and CT-based computer assisted navigational systems are currently being investigated.

Multiplanar fluoroscopy allows for real time assessment of screw placement with the unfortunate price of potential radiation exposure to the patient and surgeon and the inconvenience of the C-arm component within the surgical field. Bony anatomy can be easily defined in slender patients depending on the quality of the fluoroscopy machine. Accurate screw guidance is dependant on appropriate positioning of the $\mathrm{C}$-arm in relationship to the instrumented vertebral level. The image beam trajectory in the sagittal plane should be parallel to the superior vertebra end plate. In the transverse plane the image trajectory should be collinear to the pedicle insertion angle with the vertebral body. The spinous processes should be centered between the vertebral body boundaries to reduce any parallax effect. Once this image is obtained, a pilot hole may be made and assessed with AP fluoroscopy. Lateral images can then be obtained to assess screw path trajectory and depth.

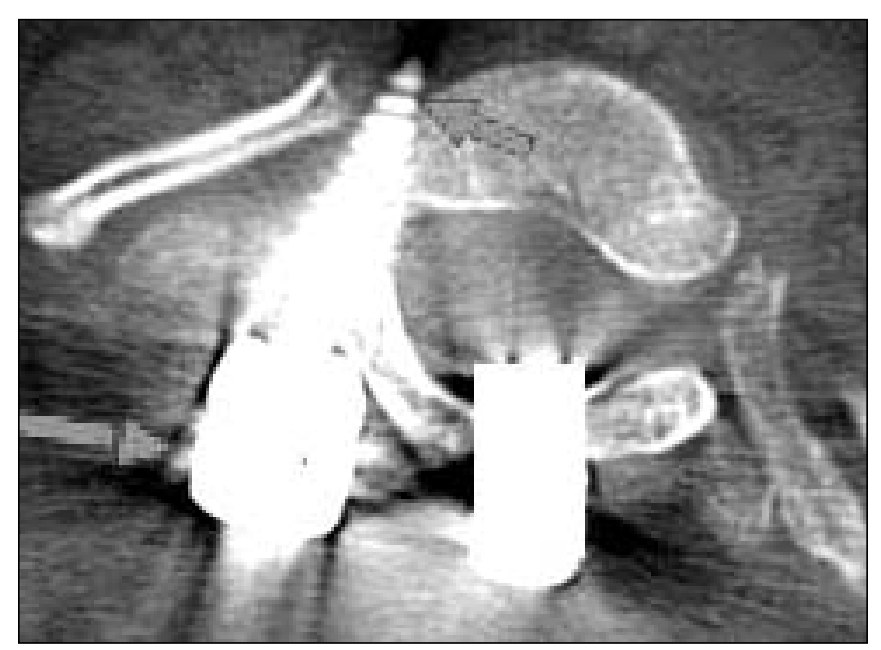

Figure 5: Axial CT image. The bottom left arrow is pointing at a prominent transverse process. Note how the screw tip was directed laterally resulting in prominence of the screw anterolaterally
There are several limitations with the use of fluoroscopy during thoracic pedicle screw placement. The quality of the images is in part dependent on the quality of the machine as well as the experience of the technologist operating the fluoroscope. An inexperienced technologist can add significant time and radiation exposure during the operative procedure. The ribs and mediastinal soft tissue may also obscure the bony anatomy in the thoracic spine during fluoroscopy. Additionally, experience is needed to understand the spatial 2D imaging presentation of the thoracic pedicles in cases of significant spinal deformity.

Carbone et a ${ }^{[6]}$ evaluated a fluoroscopically assisted technique of thoracic and thoracolumbar pedicle screw placement. Fortyone patients treated for traumatic spine injuries who underwent CT evaluation of thoracic screw placement were evaluated in a retrospective fashion. Of the 126 screws evaluated, $12.7 \%$ had penetrated the pedicle cortex with the lateral cortex being the most commonly violated ( $10.3 \%$ of all screws placed). No neurologic or visceral injuries were reported. Kuntz et $\mathrm{al}^{[7]}$ performed a prospective study evaluating the accuracy of fluoroscopically assisted pedicle screw placement. Twenty-nine patients were treated for a variety of conditions. One hundred and ninety-nine screws were reviewed with postoperative CT, $27.6 \%$ of screws were well placed within the pedicle wall boundaries. Lateral wall penetration occurred in $57.3 \%$ of screws with medial wall penetration seen in $13.6 \%$ of screws placed. No postoperative neurologic or visceral injuries were reported. There were, however, a total of six high-risk screws (five were in one patient). A highrisk screw was defined as medial pedicle wall penetration of greater than $2 \mathrm{~mm}(n=3)$ or anterolateral body penetration of greater than $3 \mathrm{~mm}$.

Recently 3D fluoroscopy has received attention in evaluating placement of thoracic pedicle screws. Wang et al ${ }^{[8]}$ evaluated horacic pedicle screw placement in a cadaveric model using the Iso-C 3D (Siemens Medical Solutions, Erlangen, Germany) fluoroscopy unit and conventional CT. Overall sensitivity and specificity was 71 and $78 \%$, respectively in detecting pedicle wall violation with the Iso-C $3 \mathrm{D}$ unit and 61 and $93 \%$, respectively using conventional CT.

Computer assisted image guided spinal surgery traditionally uses preacquired CT images downloaded onto a computer within the operating room. Defined bony landmarks are then registered intraoperatively and are visualized on the computer monitor in a multiplanar fashion. Kim et $\mathrm{al}^{[9]}$ evaluated this technique in a thoracic cadaveric model. Overall pedicle wall violation was $19.2 \%$ with major violations comprising $7.5 \%$ and minor violations comprising $11.7 \%$.

\section{Intraoperative neuromonitoring}

Intraoperative neuromonitoring (IONM) has become an integral part of complex spine surgery. The primary objective of IONM is to provide the surgeon with early warning of a potential neurological event. IONM, in the form of somatosensory-evoked potentials (SSEP's), initially gained popularity with deformity surgeons who routinely relied on the Stagnara wake up test to 
monitor neurologic integrity. Monitoring techniques have become much more sophisticated since their inception affording a higher level of protection to the patient. At present, many centers employ SSEP's, motor-evoked potentials (MEP's), and spontaneous and triggered EMG responses during complex thoracic pedicle screw instrumentation procedures.

The ideal IONM strategy would include the use of SSEP's and MEP's to monitor entire spinal cord function throughout a thoracic spinal surgery procedure. Spontaneous and triggered EMG can be used reliably in select myotomes (rectus abdominis, T8-T12) to assess the nerve roots irritability during screw hole preparation and screw placement. If MEP's are not available, SSEP's and spontaneous EMG have been used effectively as a monitoring strategy. Gunnarsson et $\mathrm{al}^{[10]}$ reported a prospective consecutive series of two hundred thoracolumbar instrumentation cases using this form of monitoring. Fourteen patients (6.6\%) awoke with new postoperative neural deficits. All of these patients had significant electomyographic activity during the procedure, however only four had significant SSEP changes. Intraoperative EMG had a $100 \%$ sensitivity and $23 \%$ specificity in detecting neural injury, whereas SSEP's had $28.6 \%$ sensitivity and $94.7 \%$ specificity. The authors concluded that the combination of these two monitoring modalities is a useful IONM strategy.

Triggered or stimulated EMG has been studied extensively in the lumbar spine to determine accurate pedicle screw placement. Lenke et $\mathrm{al}^{[11]}$ determined in a porcine model and later in a human clinical series, that a threshold stimulus of greater than $8 \mathrm{~mA}$ indicated complete intraosseous screw placement. Values of less than $4 \mathrm{~mA}$ were indicative of a pedicle wall defect. Lewis et $\mathrm{al}^{[12]}$ evaluated triggered EMG monitoring of the intercostal muscles for accuracy of thoracic pedicle screw placement in a porcine model. A definitive threshold cutoff was not able to be determined that consistently differentiated intraosseous from epidural screw placement.

A study by Reidy et al ${ }^{[13]}$ evaluated intercostal-triggered EMG in seventeen adult patients to determine accuracy of thoracic pedicle screw placement. The patients underwent postoperative CT to verify screw position. Using a cutoff value of $7 \mathrm{~mA}$, the sensitivity of intercostal-triggered EMG was $50 \%$ with a specificity of $83 \%$. The overall accuracy of the surgeon using landmarks and intraoperative imaging was $79 \%$. It was concluded by the authors that triggered EMG would not have improved the surgeons' accuracy of screw placement. ${ }^{[13]}$

\section{Discussion}

Thoracic and thoracolumbar pedicle anatomy at times may be variable depending on the presence of anatomic variants or spinal deformity making thoracic pedicle screw placement at times technically demanding. The close proximity of the spinal cord/ nerve roots and major soft tissue structures (esophagus, aorta, lung, and viscera) necessitates accurate placement of these screws to avoid potential complications. The improved biomechanical strength, usefulness in conditions where conventional hook anchorage is impossible (posterior element insufficiency), and the ability to minimize fusion levels due to predictable three-column control of the spine has popularized the use of thoracic and thoracolumbar pedicle screws over the last decade.

The question then arises: Are the advantages worth the risks associated with pedicle screw placement? Kim et $\mathrm{al}^{[5]}$ reported on the 10 years experience of Lenke and Bridwell placing pedicle screws in the thoracic spine. A total of 3204 screws were placed during this time period. Preoperative diagnoses included predominantly scoliotic and kyphotic deformities. All screws were placed using the free-hand technique. CT was utilized to randomly evaluate a total of 577 screws. In reviewing their data, $93.8 \%$ of thoracic pedicle screws were placed accurately within the bony confines of the pedicle with only $6.2 \%$ of screws demonstrating cortical perforation (1.7\% through the medial wall). No neurologic, vascular or visceral injuries were reported as a consequence of screw placement. The free-hand technique is the most technically demanding technique currently employed. A thorough understanding of the bony anatomy and experience palpating the pedicle tract are necessary for accurate screw placement. ${ }^{[14]}$

A number of surgical instruments have been implemented to successfully cannulate thoracic pedicles. The two most common instruments include a gearshift device or a cervical curette. Grauer et al. ${ }^{[15]}$ reported on the use of a novel pedicle probe the Safepath (Mekanika, Boca Raton, FL, USA). The Safepath is a nonaggressive blunt tipped drill, which is placed into the pedicle under power assistance preferentially traveling down the cancellous bone due to decreased resistance. The Safepath was initially intended for use in the lumbar spine. The Safepath device was compared to either a gearshift or cervical curette in thoracic and lumbar pedicles using a cadaveric model. The pedicles were then evaluated using plain radiography, CT, and direct inspection to detect bony violations. Upon direct inspection in the gearshift/ cervical curette group $9 \%$ of the pedicles were violated in the lumbosacral spine and $33 \%$ of the pedicles in the thoracic spine were violated. On direct inspection of the Safepath group there were no pedicle violations in the lumbosacral group, however, $76 \%$ of the thoracic pedicles were violated. The authors concluded the Safepath was efficacious in the lumbar spine but was significantly worse than traditional methods in the thoracic spine.

Issues have been raised regarding the safety of thoracic pedicle screw placement in the setting of significant spinal deformity, especially in the pediatric population. Suk et al. ${ }^{[16]}$ evaluated the placement of 4604 thoracic pedicle screws to correct spinal deformity. Suspicious screws on postoperative radiographs were evaluated with CT. Sixty-seven (1.5\%) malpositioned screws were noted. There was one case of a transient paraparesis due to medial screw perforation. The screw was removed and the patient made a complete recovery 3 weeks later. The deformity correction obtained in the idiopathic scoliosis group averaged $66.3 \%$ at final followup.

Kuklo et al. ${ }^{[17]}$ reported on the safety and efficacy of thoracic pedicle screws in patients with coronal deformities greater than 
$90^{\circ}$. Twenty patients that met this criteria had a total of 352 thoracic pedicle screws placed and were followed for an average of approximately 3 years. Intrapedicular screw placement or a breach of less than two millimeters was reported in $96.3 \%$ of screws determined by postoperative CT imaging. There were three screws that had a breach of greater than four millimeters and the two medial screws were subsequently removed. There were no visceral or neurologic injuries reported due to screw placement. The authors concluded that even in large magnitude curves that thoracic pedicle screws can be placed effectively.

The placement of thoracic pedicle screws in the pediatric population for idiopathic scoliosis imposes unique challenges. The pedicles on the concavity of the deformity, typically on the left, tend to be smaller and more sclerotic. In addition, the spinal cord is closer in proximity to the pedicles on the concave side of the curve due to the rotational deformity. A thorough understanding of the 3D bony anatomy is critical to ensure accurate thoracic screw placement, especially when instrumenting the left side of the thoracic spine due to the close proximity of the aorta to the screw path.

Intraoperative imaging can assist a surgeon in the placement of thoracic pedicle screws. With the use of intraoperative imaging, pedicle wall breaches have been reported to range between 3.7 and $38.9 \% .^{[9,18-21]}$ Using bony landmarks alone, pedicle wall breaches have been reported in the range of $15.9-54.7 \% .^{[2,3,18,22]}$ Plain radiography is available in all operating theatres during spinal instrumentation procedures, however it only provides a static image of an intraoperative event and repeat films following each step in an instrumentation procedure is extremely time consuming. Odgers et al. ${ }^{[20]}$ studied the accuracy of intraoperative lateral plain radiographs when placing thoracolumbar pedicle screws. Screws were evaluated postoperatively with CT and found to be within the pedicle boundaries in $89.1 \%$ of screws placed.

Multiplanar fluoroscopy is the most commonly used real time imaging technique employed by surgeons to assist in the placement of thoracic pedicle screws. Most hospitals have recent generation fluoroscopes, which provide detailed bony anatomy. Advantages of traditional C-arm technologies include easy intraoperative maneuverability, ability to image in multiple planes, and the familiarity of most surgeons with this imaging technology. Additionally, the length and trajectory of the screw can be appropriately guided with this method of imaging. Screw trajectory is especially important in terms of spinal biomechanics as demonstrated by Lehman et al. ${ }^{[23]}$ In their study, the straight on screw trajectory path provided a higher pull-out strength compared to following the anatomic trajectory of the native pedicle. Potential issues with increased infection rates while using $\mathrm{C}$-arm have not been borne out in the literature, however, real concerns exist regarding potential excessive radiation exposure to both the surgical staff and patient during screw placement. ${ }^{[6]}$

The surgeon and the supporting operating room staff can take protective measures to minimize their exposure to the ionizing radiation associated with fluoroscopy, however what is the exposure to the unprotected patient? Perisinakis et al. ${ }^{[24]}$ studied the estimated radiation dose a patient receives during the placement of fluoroscopically guided pedicle screws (T11-L5). The average fluoroscopy time was $1.2 \mathrm{~min}$ in the AP plane and $2.1 \mathrm{~min}$ in the lateral plane. The average gonadal dose in female patients was $0.67 \mathrm{~m}$ Gy and for male patients $0.12 \mathrm{~m}$ Gy. The average radiogenic risk for fatal cancer and genetic defects was 115 and 4 per million patients treated for women and men, respectively. The use of fluoroscopy in the lateral plane is associated with higher levels of radiation exposure due to the greater soft tissue penetration required to obtain images. In addition, patients with a larger body habitus will require higher energy levels to achieve adequate visualization of the bony anatomy thus resulting in higher levels of radiation exposure.

The use of fluoroscopy and exposure to ionizing radiation is also a major concern to the spine surgeon. As previously mentioned surgeons can protect themselves with eyewear, thyroid shields, and lead aprons. As a result the surgeon's hands are at the highest risk of radiation exposure. Rampersaud et al. ${ }^{[25]}$ evaluated the amount of radiation exposure to the surgeon's neck, torso, and dominant hand during placement of pedicle screws from T11S1 using lateral fluoroscopy only in a cadaveric model. Average fluoroscopy exposure time was 9.3 s per screw. The average hand dose rate was $58.2 \mathrm{mrem} / \mathrm{min}$. The internationally recommended maximum limit for annual hand radiation exposure is 50,000 mrem. ${ }^{[26,27]}$ A significant increase in hand dose rate was noted when placement of the screw was on the same side of the beam source as well as when a heavier cadaver was imaged. The authors noted that the use of radiation attenuation gloves reduced the hand dose rate by $33 \%$. When the authors compared their data to other studies spine surgeons had a 10-12-fold increase in hand radiation exposure compared with other musculoskeletal extremity procedures. There are several reasons for this finding. First, there are typically multiple levels that will be instrumented during the spinal procedure, which in itself lends to more exposure. Second, the bilaterality of spinal procedures requires the surgeon to stand on the side that has the beam source, which results in a larger exposure as demonstrated in this study. Finally, the thickness of the soft tissues in the thoracic and thoracolumbar spines require higher energy levels to achieve visualization of the bony anatomy which results in higher radiation exposure.

Advanced imaging techniques, including 3D fluoroscopy and CT-based computer navigational systems, have not been readily incorporated into current spine practices mainly because of cost issues. 3D fluoroscopy requires acquisition times on the order of minutes to evaluate instrumented levels. With regard to CT-base computer navigational systems, data registration has been an issue. Registration errors on the order of millimeters can have significant impact when cannulating the narrow thoracic pedicles when the average pedicle diameter is $4.5 \mathrm{~mm}$ at $\mathrm{T} 4{ }^{\left[{ }^{[28]}\right.}$ These techniques will require further evaluation to assess their role in facilitating the accuracy of placing thoracic pedicle screws.

The critical evaluation of postoperative plain films is mandatory. Screws should be evaluated for length, position in relation to the pedicle, and their relationship to surrounding screws. Any screw 
that appears to cross the midline on the AP film should raise concern for a medial pedicle wall perforation and should be further evaluated by CT. Furthermore, any suspicious screw noted on postoperative plain films warrants examination by CT to confirm placement (Figure 6). Screws with lateral (most common) or medial penetration and without a neurologic deficit most likely do not need screw placement revision provided they have adequate bony purchase. Screw redirection should be considered for screws that penetrate the anterior vertebral body due to the close proximity to the aorta, lung, and intrathoracic viscera. Fortunately, aortic injury is extremely rare with a paucity of cases being reported in the literature. ${ }^{[29,30]}$ Caution must be exerted with interpretation of postoperative CT. Accuracy of CT in assessing thoracic pedicle screw malposition has been studied in a cadaveric model by Fayyazi $^{[31]}$ et al. CT, when compared to open inspection, had a sensitivity of $71 \%$, specificity of $75 \%$ and accuracy of $76 \%$. The authors concluded that in the face of a new neurologic deficit, or great vessel or lung injury, CT may not be sufficient to assess safe screw position and surgical exploration may be warranted. Rao et al. ${ }^{[32]}$ performed a study to validate the use of CT as a means of assessing the accuracy of thoracic pedicle screw placement. One hundred and fifty-five pedicles were instrumented in a cadaveric model. The screws were evaluated with $\mathrm{CT}$ and by direct visualization. A kappa value of 0.51 was determined which depicted a moderate level of agreement between the two measurement techniques. CT was found to have a sensitivity of $86 \%$ and specificity of $85 \%$ for detecting accuracy of screw placement. Most importantly the authors found a negative predictive value of $62 \%$. The authors concluded that $\mathrm{CT}$ is useful in assessing screw placement, however, it tends to overestimate screw misplacement.

The placement of thoracic pedicle screws offers many biomechanical advantages over conventional hook and rod constructs including increased pull-out strength and maintenance of deformity correction. The literature supports that this type of instrumentation can be placed safely in a variety of clinical

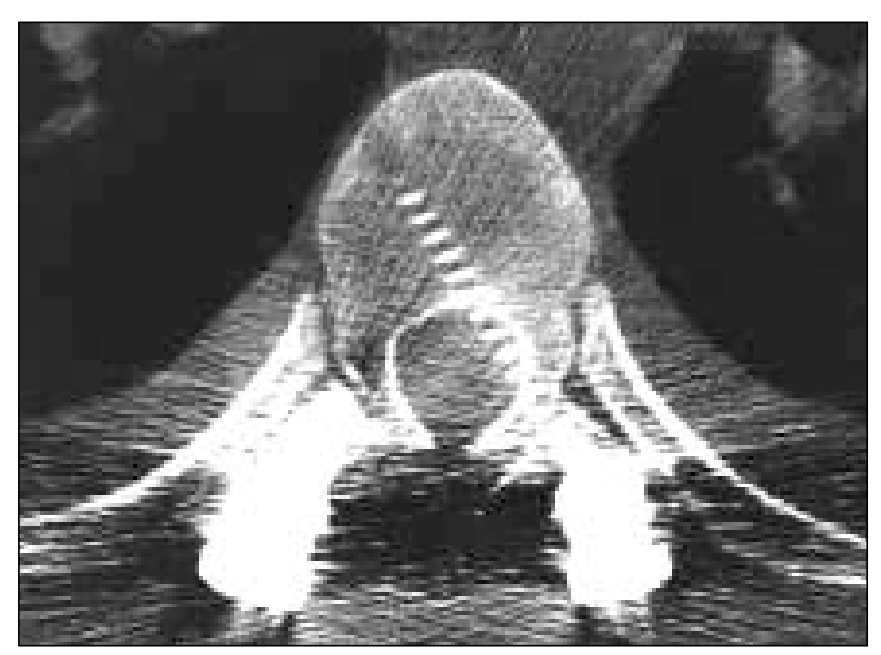

Figure 6: Axial CT image demonstrating a medially malpositioned thoracic pedicle screw conditions with minimal reports of serious neurologic or visceral injuries. The additional risk of radiation exposure to the patient and the surgeon are acceptable when fluoroscopy is used appropriately. Surgeons should use only pulse shots of fluoroscopy to minimize the overall radiation exposure to the patient and themselves. The surgeon should keep their hand out of the beam trajectory by initially cannulating the pedicle to a minimal depth then leaving the gearshift or curette in the bone and removing their hand from the field while imaging. Finally, the surgeon must be aware of their fluoro use during the case and use it only as necessary.

Surgeons that either lack experience or the appropriate technology should be hesitant to embark on placement of this type of instrumentation. Surgeons should only proceed with thoracic pedicle screws once they have mastered the placement of lumbar pedicle screws. In countries lacking in sophisticated operating theatre technology it may not be technically feasible to perform these procedures. A fluoroscopy machine that can provide adequate $\mathrm{AP}$ and lateral images is all that is needed from a radiology standpoint for placement of thoracic pedicle screws. Surgeons while introducing this technique into their practice can confirm their intraoperative knowledge of the spinal anatomy with the fluoroscopic images. As surgeons becomes more comfortable they can define the starting points from the bony anatomy alone using AP fluoroscopy sparingly and relying on lateral fluoroscopy for guiding screw trajectory. Certainly, the type of cases in which the surgeon chooses to place thoracic pedicle screw instrumentation affects the complexity of the procedure. Initially, trauma and tumor cases maybe less challenging because of the lower likelihood of significant coronal imbalance.

\section{Conclusion}

Thoracic and thoracolumbar pedicle screw instrumentation is proving to be a safe and reliable method of obtaining rigid segmental fixation of the thoracic spine. A thorough understanding of the complex 3D spinal anatomy is required to safely place this type of instrumentation. The biomechanical benefits that are derived from using pedicle screw instrumentation in all forms of spinal pathology are the driving force behind more and more surgeons incorporating thoracic and thoracolumbar pedicle screw placement into their practices. Surgeons however, must be well versed in the placement of complex spinal instrumentation in order to accurately and safely use this method of instrumentation in all types of spinal disorders.

\section{References}

1. Suk SI, Lee CK, Min HJ, Cho KH, Oh JH. Comparison of Cotrel-Dubousset pedicle screws and hooks in the treatment of idiopathic scoliosis. Int Orthop 1994;18:3416.

2. Liljenqvist UR, Halm HF, Link TM. Pedicle screw instrumentation of thoracic spine in idiopathic scoliosis. Spine 1997;22:2239-45.

3. Vaccaro AR, Rizzolo SJ, Balderston RA, Allardyce T.J, Garfin SR, Dolinskas C, et al. Placement of Pedicle Screws in the Thoracic Spine. Part II: An Anatomical and Radiographic Assessment. J Bone Joint Surg Am 1995;77:1200-6.

4. Sucato DJ, Duchene C. The position of the aorta relative to the spine: a comparison of patients with and without idiopathic scoliosis. J Bone Joint Surg Am 
2003;85:1461-9.

5. Kim Y.J, Lenke LG, Bridwell KH, Cho YS, Riew KD. Free Hand Pedicle Screw Placement in the Thoracic Spine: Is it Safe? Spine 2004;29:333-42.

6. Carbone JJ, Tortolani PJ, Quartararo LG. Fluoroscopically assisted pedicle screw fixation for thoracic and thoracolumbar injuries. Technique and short-term complications. Spine 2003;28:91-7.

7. Kuntz C 4th, Maher PC, Levine NB, Kurokawa R. Prospective evaluation of thoracie pedicle screw placement using fluoroscopic imaging. J Spinal Disord Tech 2004;17:206-8.

8. Wang MY, Kim KA, Liu CY, Kim P, Apuzzo ML. Reliability of Three-Dimensional Fluoroscopy for Detecting Pedicle Screw Violations in the Thoracic and Lumbar Spine. Neurosurgery 2004;54:1138-42

9. Kim KD, Patrick .J.J, Bloch BS, Masciopinto .JE. Computer-assisted thoracic pedicle screw placement: An in vitro feasibility study. Spine 2001;26:360-4

10. Gunnarsson T, Krassioukov AV, Sarjeant R, Fehlings MG. Real-time continous intraoperative electromyographic and somatosensory evoked potential recordings in spinal surgery: correlation of clinical and electrophysiologic findings in a prospective, consecutive series of 213 cases. Spine 2004;29:677-84

11. Lenke LG, Padberg AM, Russo MH, Bridwell KH, Gelb DE. Triggered electromyographic threshold for accuracy of pedicle screw placement. An animal model and clinical correlation. Spine 1995;20:1585-91.

12. Lewis SJ, Lenke LG, Raynor B, Long J, Bridwell KH, Padberg A. Triggered Electromyographic Threshold for Accuracy of Thoracic Pedicle Screw Placement in a Porcine Model. Spine 2001;26:2485-9.

13. Reidy DP, Houlden D, Nolan PC, Kim M, Finkelstein JA. Evaluation of Electromyographic Monitoring During Insertion of Thoracic Pedicle Screws. J Bone Joint Surg Br 2001;83:1009-14.

14. Lehman RA, Potter BK, Kuklo TR, Chang AS, Polly DW, Shawen SB, et al. Probing for thoracic pedicle screw tract violation(s). Is it valid? J Spinal Disord Tech $2004 ; 17: 277-83$.

15. Grauer JN, Vacearo AR, Brusovanik G, Girardi FP, Silveri CP, Cammisa FP, et al. Evaluatiion of a novel pedicle probe for the placement of thoracic and lumbosacral screws. J Spinal Disord Tech 2004;17:492-7.

16. Suk SI, Kim WJ, Lee SM, Kim JH, Chung ER. Thoracic Pedicle Screw Fixation in Spinal Deformities: Are They Really Safe? Spine 2001;26: 2049-57.

17. Kuklo TR, Lenke LG, O'Brien MF, Lehman RA Jr, Polly DW Jr, Schroeder TM. Accuracy and Efficacy of Thoracic Pedicle Screws in Curves More Than 90 degrees. Spine 2005;30:222-6.

18. Belmont PJ Jr, Klemme WR, Dhawan A, Polly DW Jr. In vivo Accuracy of Thoracic
Pedicle Screws. Spine 2001;26:2340-6.

19. Merloz P, Tonetti .J, Pittet L, Coulomb M, Lavallee S, Sautot P. Pedicle Screw Placement Using Image Guided Techniques. Clin Orthop Relat Res 1998;354:39-48.

20. Odgers C.J 4th, Vaccaro AR, Pollack ME Cotler JM Accuracy of pedicle screw placement with the assistance of lateral plain radiography. J Spinal Disord Tech 1996;9:334-8

21. Gertzbein SD, Robbins SE. Accuracy of pedicular screw placement in vivo. Spine $1990 ; 15: 11-4$

22. Xu R, Ebraheim NA, Ou Y, Yeasting RA. Anatomic considerations of pedicle screw placement in the thoracic spine: Roy-Camille technique versus open lamina technique. Spine 1998;23:1065-8.

23. Lehman RA Jr, Polly DW Jr, Kuklo TR, Cunningham B, Kirk KL, Belmont P.J .Jı: Straight-forward versus anatomic trajectory technique of thoracic pedicle screw fixation: a biomechanical analysis. Spine 2003;28:2058-65.

24. Perisinakis K, Theocharopoulos N, Damilakis .J, Katonis P, Papadokostakis G, Hadjipavlou A, et al. Estimation of patient dose and associated radiogenic risks from fluoroscopically guided pedicle screw insertion. Spine 2004;29:1555-60.

25. Rampersaud YR, Foley KT, Shen AC, Williams S, Solomito M. Radiation Exposure to the Spine Surgeon During Fluroscopically Assisted Pedicle Screw Insertion. Spine 2000;25:2637-45.

26. Recommendations on limits for exposure to ionizing radiation. National council on Radiation Protection and Measurements. Bethesda, MD: 1987. p. 91.

27. Recommendations of the International Commission on Radiological Protection International Commission on Radiological Protection, Publication 60. 1991. p. 21

28. Vaccaro AR, Rizzolo S.J, Allardyce T.J, Ramsey M, Salvo J, Balderston RA, et al Placement of Pedicle Screws in the Thoracic Spine: Part 1: Morphometric Analysis of the Thoracic Vertebrae. J Bone Joint Surg Am 1995;77:1193-9.

29. Minor ME, Morrissey N.J, Peress R, Carroccio A, Ellozy S, Agarwal G, et al. Endovascular treatment of an iatrogenic thoracic aortic injury after spinal instrumentation: a case report. J Vase Surg 2004;39:893-6.

30. Vanichkachorn JS, Vaccaro AR, Cohen MJ, Cotler JM. Potential large vessel injury during thoracolumbar pedicle screw removal. A case report. Spine 1997;22:110-3.

31. Fayyazi AH, Hugate RR, Pennypacker J, Gelb DE, Ludwig SC. Accuracy of computed tomography in assessing thoracic pedicle screw malposition. J Spinal Disord Tech 2004;17:367-71.

32. Rao G, Brodke DS, Rondina M, Dailey AT. Comparison of computerized tomography and direct visualization in thoracic pedicle screw placement. J Neurosurgery $2002 ; 97: 223-6$ 